\title{
IMPLANTATION IN OVARIECTOMIZED MICE TREATED WITH DIBUTYRYL ADENOSINE 3',5'- MONOPHOSPHATE (DIBUTYRYL CYCLIC AMP)
}

\author{
F. T, G. WEBB* \\ Department of Zoology, South Parks Road, Oxford OX1 3PS
}

(Received 3rd August 1974)

\begin{abstract}
Summary. Experiments are described that demonstrate that uterine intraluminal injection of a $1.25 \mathrm{~mm}$-solution of dibutyryl cyclic AMP (dcAMP) in phosphate buffered saline (PBS) induced implantation in ovariectomized pregnant mice. Pretreatment with progesterone was essential for this effect. When PBS was injected alone, it did not induce implantation in mice treated with progesterone. Bilateral adrenalectomy had no effect on the ability of dcAMP to substitute for oestradiol, showing that the effect was not due to dcAMP-induced oestrogen synthesis in the adrenal cortex. It is suggested that the dcAMP may act at the level of the uterus, the embryo, or both.
\end{abstract}

\section{INTRODUCTION}

There is considerable evidence to show that in the ovariectomized mouse and rat, treatment with progesterone alone is insufficient for the implantation of embryos to occur. When, however, a discrete dose of oestrogen is given after pretreatment for at least 2 days with progesterone, the embryos implant and develop normally both in the mouse (Humphrey, 1967; Smith \& Biggers, 1968) and the rat (Mayer, 1963).

Adenosine 3',5'-monophosphate (cyclic AMP) has been shown to mimic some of the early anabolic effects of oestradiol in the rat uterus (Hechter, Yoshinaga, Halkerston \& Birchall, 1967; Griffin \& Szego, 1968; Mohla \& Prasad, 1970). The studies reported here were carried out to determine whether cyclic AMP could substitute for the nidatory action of oestradiol in the mouse.

\section{MATERIALS AND METHODS}

Virgin female mice between 6 and 9 weeks of age from a closed colony of the A2G strain in the Department of Zoology, Oxford, were caged with males of the same strain and checked each day for the presence of a vaginal plug. When found, the plug was taken to indicate Day 1 of gestation. All mice were ovariectomized during the morning of Day 3 and were left without treatment

* Present address: Physiological Laboratory, Downing Street, Cambridge CB2 3EG. 
for Days 4, 5 and 6. Thereafter, treatment varied depending on the experimental group to which the mice were assigned. The period without treatment allowed implantation induced during subsequent exogenous treatment to be distinguished readily from implantations at the normal time as a result of, for example, incomplete ovariectomy. From Day 7 until autopsy, the mice in Groups 1 to 4 received daily subcutaneous injections of $1 \mathrm{mg}$ progesterone (Koch-Light Laboratories) in $0.05 \mathrm{ml}$ maize oil. The mice in Group 1 received no other treatment whereas those in Group 2 received daily subcutaneous injections of $20 \mathrm{ng}$ oestradiol-17 $\beta$ (Sigma) in $0.05 \mathrm{ml}$ maize oil, from Day 9 (3rd day of progesterone treatment) until autopsy. The mice in Group 3 were given a single uterine intraluminal injection of $0.01 \mathrm{ml}$ of a $1.25 \mathrm{~mm}$-solution of dibutyryl cyclic AMP (dcAMP, Sigma) in phosphate buffered saline (PBS: Dulbecco \& Vogt, 1954) on Day 9 followed by daily intraperitoneal injections of 0.04 $\mathrm{ml}$ of the same solution until autopsy. The mice in Group 4 were treated as those in Group 3 except that PBS alone was given to the mice treated with progesterone.

The mice in Groups 5 and 6 were not given progesterone but received injections of dcAMP only, those in Group 5 having intraperitoneal injections from Day 7 until autopsy with a single uterine intraluminal injection on Day 9 and those in Group 6 having intraperitoneal injections from Day 8 and intraluminal injections on Days 7 and 9.

Mice in Group 7 were treated as those in Group 3 except that bilateral adrenalectomy was carried out through a mid-dorsal incision immediately before the uterine intraluminal injection of dcAMP. The technique for this operation was developed from the methods of Grollman (1941) and Llaurado (1958) and afterwards the mice were kept in an incubator at $31^{\circ} \mathrm{C}$ with $1 \%$ saline to drink (Grollman, 1941). At autopsy, mice were checked for residual pieces of adrenal, but none was found.

The dcAMP was used because it has been reported to exhibit greater penetration of cells and greater resistance to hydrolysis by phosphodiesterase than cyclic AMP (Posternak, Sutherland \& Henion, 1962). Intraluminal injection was always achieved through the uterotubal junction, although not always from the same side.

At autopsy on Day 11, 12, 13 or 14, uteri with implantation sites were fixed in Bouin's fluid and embedded in Fibrowax (R. A. Lamb, Ltd, 8 Sunbeam Rd, London NW10). Most, but not all, of the decidual swellings were sectioned at $8 \mu \mathrm{m}$ and stained in Mayer's haemalum and light green. The stage of embryonic development was estimated by comparison with embryos of known age taken from intact pregnant mice and with reference to Snell \& Stevens (1966) and Rugh (1968). The normality of embryonic development was assessed using the criteria of orientation of the embryo with respect to the uterus, differentiation of the trophoblast and inner cell mass, breakdown of the uterine epithelium, invasion of the trophoblast and formation of the embryonic germ layers and membranes.

If implantation was not observed, the uterine horns were flushed separately with PBS and the flushings were searched for embryos.

Student's $t$ test was used to establish the significance of the difference 
between two means. Values of $P$ less than 0.05 were taken as significant. The significance of the difference between two percentages was established using the $\chi^{2}$ test.

\section{RESULTS}

The results of the experiments are presented in Table 1 . In mice receiving progesterone treatment alone, no implantations were seen at laparotomy on Day 15 or at autopsy on Day 20. Daily injections of oestradiol from Day 9 resulted in implantation in $58 \%$ of the animals that had been pretreated with progesterone. Only one mouse showed a bilateral response and the mean number of implantations per mouse was $2 \cdot 7$. Uterine intraluminal injection of dcAMP on Day 9 followed by daily intraperitoneal injections (Group 3) induced implantation in $59 \%$ of animals. The difference between these two percentages was not significant $(P=0 \cdot 25)$. Seven out of thirteen mice in Group 3 showed a bilateral response and a further three had implantations in the contralateral (non-injected) horn only. The mean number of implantations/ mouse was $3 \cdot 8$.

When PBS was administered instead of dcAMP (Group 4), no implantation resulted, although blastocysts could be flushed from the uteri. Blastocysts did not implant when dcAMP was given alone without concurrent progesterone treatment (Groups 5 and 6).

Bilateral adrenalectomy appeared to have no effect on the ability of dcAMP to mimic the nidatory action of oestradiol (Group 7) with regard to the number of animals responding $(66 \%)$ and to the mean number of implantations/mouse $(2 \cdot 4)$. The effect was bilateral in two out of twelve animals; six mice had implantations in the contralateral horn only.

All except two of the implantation sites examined histologically contained developing embryos. Most embryos appeared in sagittal section which is normal in transverse sections of the uterus (Snell \& Stevens, 1966), although two were found to be sectioned transversely. This is an unusual observation and appears to have no clear explanation.

All of the embryos implanting following injection of oestradiol were developing normally. Development resulting from dcAMP-induced implantation (Plate 1) was generally comparable, morphologically, to that seen in embryos implanting after treatment of the mother with oestradiol. In one case, however, the embryo was dying and beginning to be resorbed. Decidualization occurred normally as did the regression of the epithelium in the vicinity of the implanting embryo. Bilateral adrenalectomy had no effect on the normality of embryonic development.

Day 9 of pregnancy in oestradiol-treated mice appeared to correspond to the 4th day of normal pregnancy since embryos in the uterus at autopsy on Day 11 were at a stage comparable to that seen on the 6th day of normal pregnancy. Similarly, on Days 12, 13 and 14, embryonic development was comparable to that at the 7th, 8th and 9th days of gestation. A feature of development resulting from dcAMP treatment, particularly when autopsy was on Day 13 or 14, was that the embryos were often 1 day behind this 'expected stage of development'. In no case was development sufficiently advanced to suggest that 


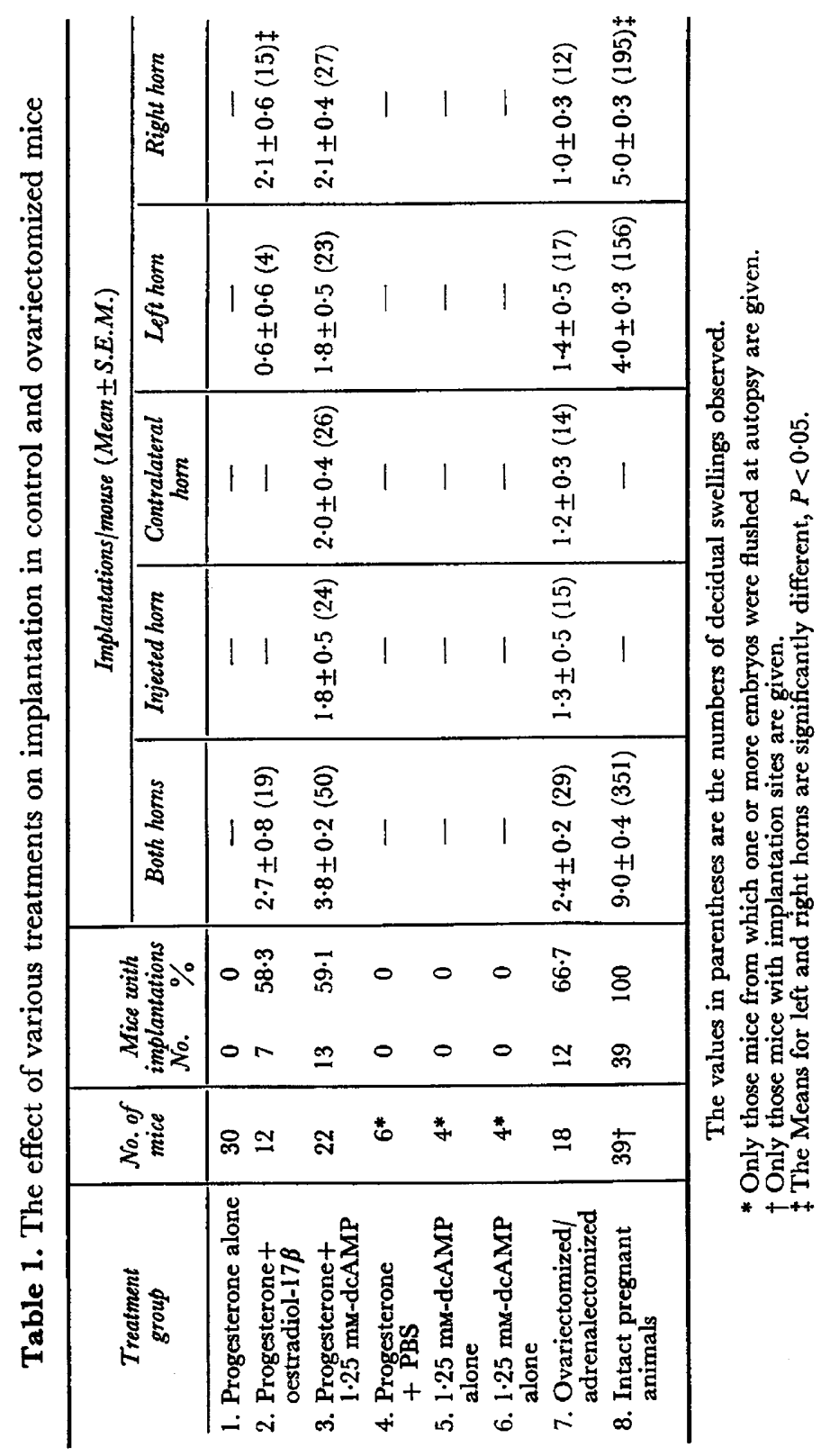


PLATE 1

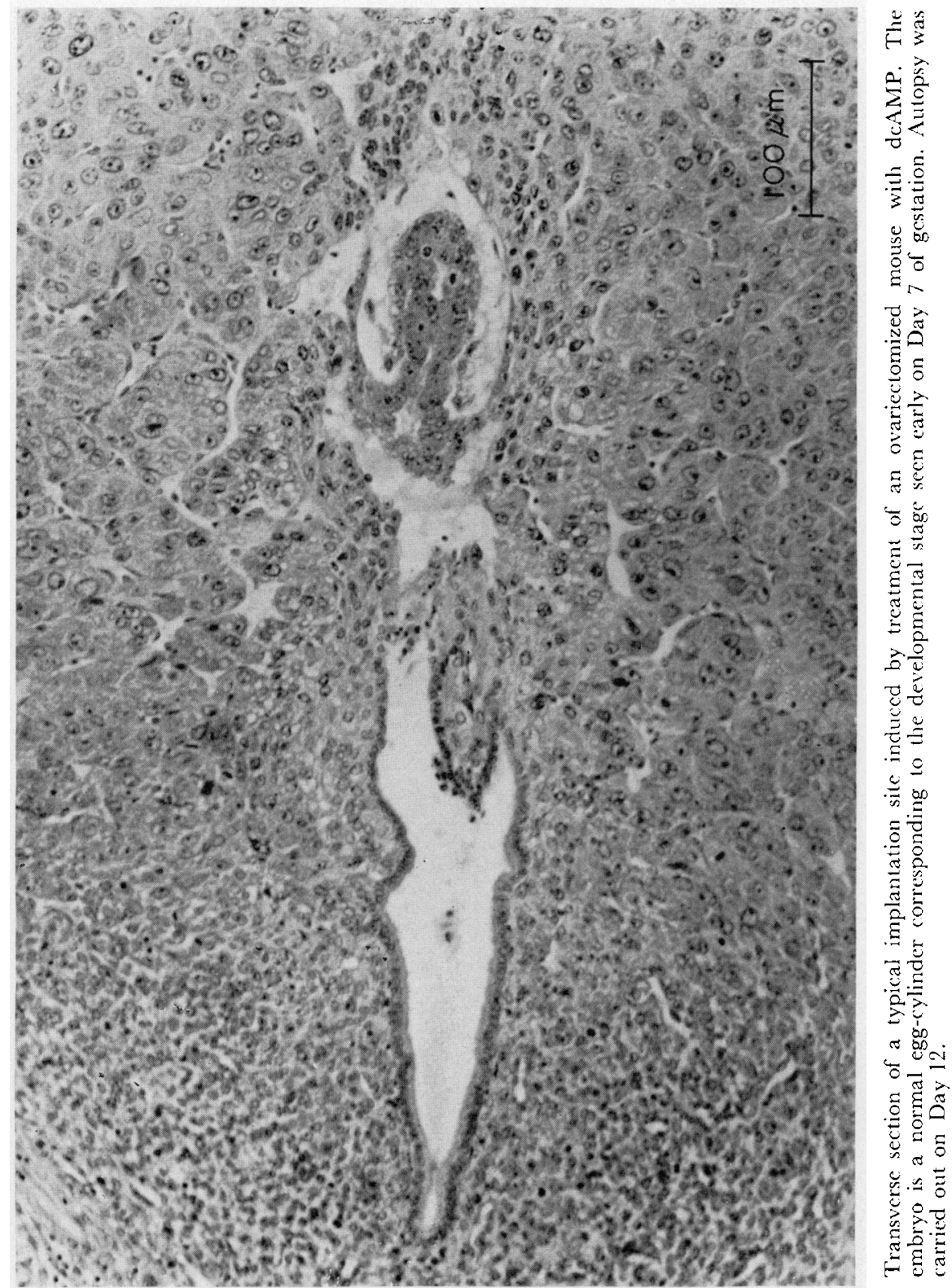

(Facing p. 514) 
implantation had begun before the administration of either oestradiol or dcAMP.

\section{DISCUSSION}

Humphrey (1967) and Smith \& Biggers (1968) have shown that blastocysts in the uteri of ovariectomized mice treated with progesterone will only implant following exogenous administration of oestrogen. This result was confirmed in the present work where it was also demonstrated that instillation of dcAMP into the uterus of a mouse deprived of oestrogen induced implantation and normal development of blastocysts.

In preliminary experiments with progesterone-treated mice, to which only the uterine intraluminal injection of dcAMP was given, implantation occurred but development did not reach the egg-cylinder stage. Intraperitoneal injections given after the intraluminal injection supported development for longer, although intraperitoneal injections alone were unable to induce implantation.

Pretreatment with progesterone was essential for implantation to be induced with dcAMP. Blastocysts did not implant following intraluminal administration of PBS alone showing that the response was not due to substances released from damaged cells as a result of the trauma of the injection. Although implantation has been observed following administration of more than $4.0 \mathrm{mg}$ Koch-Light progesterone (P. S. Grant, personal communication), progesterone treatment alone was without effect at the dose level given in the present experiments.

There are several ways in which the apparent substitution of dcAMP for oestrogen may be explained. Firstly, dcAMP may stimulate the synthesis or release of oestrogen in extraovarian sites. Some support for a systemic action of dcAMP comes from the observation that following the unilateral injection of dcAMP, a bilateral effect was often seen. Furthermore, the development of many of the embryos implanting as a result of dcAMP treatment was as much as $24 \mathrm{hr}$ behind that of embryos implanting following the injection of oestradiol, a delay that might have resulted from the time taken to stimulate steroid-producing or steroid-storing tissues elsewhere in the body. The speedy hydrolysis of cyclic AMP by phosphodiesterase (Cheung, 1970) would suggest that this is unlikely, although the dibutyryl analogue of cyclic AMP used in these experiments is more resistant to the action of phosphodiesterase (Posternak et al., 1962).

Steroids are produced in the ovaries and in the adrenal cortex of the female mouse and it has long been suggested that the adrenals are a second source of both progesterone and oestrogen (see Short, 1960, for references and discussion). The rôle of cyclic AMP in adrenal steroidogenesis is not completely understood (see Berger, 1971) and it may be, therefore, that the dcAMP used in these experiments was causing the normally minor pathway of oestrogen synthesis to become a major one. This possibility can probably be excluded on theoretical grounds (Webb, 1973) as although low intracellular levels of cyclic AMP in the rat adrenal cortex are known to stimulate the synthesis of pregnenolone from cholesterol, the evidence suggests that high levels may inhibit the 
overall synthesis of steroids by inhibition of the synthesis of progesterone from pregnenolone (Berger, 1971). In any case, ovariectomized mice subjected to bilateral adrenalectomy immediately before the instillation of dcAMP showed a bilateral implantation response at subsequent autopsy (Table 1). Takahashi (1961) has reported oestrogen retention by several organs of the rabbit following ovariectomy and adrenalectomy so, in the present study, dcAMP-induced release of stored oestrogen from the tissues of mice cannot be ruled out.

The most likely explanation for the bilateral effect, however, is that although only $0.01 \mathrm{ml}$ dcAMP solution was injected, some of the solution reached the contralateral horn. In the mouse, there is only one cervical canal so that the two uterine lumina are confluent (Leppi, 1964; Hummel, Richardson \& Fekete, 1966), although the degree of contiguity appears to depend on the strain of mouse (Bartke, 1968). In the strain used in the present experiments, $0.01 \mathrm{ml}$ of a solution of pontamine blue in PBS, but not of arachis oil, can be seen to migrate to the contralateral horn within a few minutes of unilateral injection through the uterotubal junction (F. T. G. Webb, unpublished observation). A solution of dcAMP in PBS, having a similar viscosity to the pontamine blue solution, is likely to have done the same.

There is no clear explanation for the retarded development of embryos in uteri treated with dcAMP compared with those treated with oestradiol. The dose level of dcAMP may not have been the most appropriate, however, as treatment with 2.5 or $3.75 \mathrm{~mm}$-dcAMP has been observed often to result in development at a rate comparable to that shown by embryos in uteri treated with oestradiol (F. T. G. Webb, unpublished results).

In inducing implantation, the dcAMP may have been acting only locally. If this is the case, then the results imply a rôle for cyclic AMP in egg implantation in mice, at the level of the uterus, the embryo or both. A number of workers have demonstrated the ability of cyclic AMP to mimic the anabolic effects of oestradiol on the uteri of castrated rats (Hechter \& Soifer, 1971). These observations have led to the suggestion that cyclic AMP may be involved in the action of oestrogen at the level of the genome, although this is in dispute (see Hechter \& Soifer, 1971). It is not possible to comment on this idea from the results reported in the present work as the site of action of the dcAMP is not known. Whatever the nature of this mimicry of oestrogen action, however, it is possible that if dcAMP is able to reproduce the required oestrogenic effect on the progestational uterus (Finn, 1966, 1971), sensitization will occur. This possibility has been the subject of further research.

Alternatively, dcAMP may exert effects on the embryo (Mohla \& Prasad, 1970), either substituting for a direct effect of oestrogen or mimicking the action of a compound which activates the embryo by stimulation of adenyl cyclase and which is normally produced in uterine tissue in response to oestrogen. Possible effects of dcAMP on the preimplantation mouse embryo are being investigated.

\section{ACKNOWLEDGMENTS}

This work was carried out during the tenure of a NATO Research Studentship from the Science Research Council. The author is indebted to Dr Christopher 
Graham for his constructive criticism and advice. Some of the histological work was done by Mrs Zillah Deussen.

\section{REFERENGES}

BARTKE, A. (1968) Effect of an IUD on implantation and the decidual reaction in different strains of mice. F. Reprod. Fert. 15, 185-190.

Berger, G. M. B. (1971) Possible role of cyclic AMP in the short term regulation of adrenal steroidogenesis. Nature, Lond. 232, 474-475.

Gheung, W. Y. (1970) Gyclic nucleotide phosphodiesterase. In Role of Cyclic AMP in Cell Function, pp. 51-66. Eds. P. Greengard and E. Costa. Raven Press, New York.

Dulbecco, R. \& Vogt, M. (1954) Plaque formation and isolation of pure lines with poliomyelitis virus. 7. exp. Med. 99, 167-182.

FinN, G. A. (1966) Endocrine control of endometrial sensitivity during the induction of the decidual reaction in the mouse. F. Endocr. 36, 239-248.

Fins, G. A. (1971) Biology of decidual cells. Adv. Reprod. Physiol, 5, 1-26.

Griffin, D. M. \& Szego, C. M. (1968) Cyclic AMP stimulation of uterine amino acid uptake in vitro. Life Sci. 7, 1017-1023.

Grollman, A. (1941) Biological assay of adrenal cortical activity. Endocrinology, 29, 855-861.

HeChter, O. \& Solfer, D. (1971) Involvement of the adenyl cyclase $3^{\prime}, 5^{\prime}$-AMP system in steroid hormone action. In Basic Action of Sex Steroids on Target Organs, pp. 93-111. Eds. P. O. Hubinont, F. Leroy and P. Galand. Karger, Basel.

Hechter, O., Yoshinaga, K., Halkerston, I. D. K. \& Birchall, K. (1967) Estrogen-like anabolic effects of cyclic $3^{\prime}, 5^{\prime}$-adenosine monophosphate and other nucleotides in isolated rat uterus. Archs Biochem. Biophys. 122, 449-465.

Hummel, K. P., Richardson, F. L. \& Fekete, E. (1966) Anatomy. In Biology of the Laboratory Mouse, 2nd edn, pp. 247-307. Ed. E. L. Green. McGraw-Hill, London.

HuMphrey, K. W. (1967) The induction of implantation in the mouse after ovariectomy. Steroids, 10, $591-600$.

LePpi, T. J. (1964) A study of the uterine cervix of the mouse. Anat. Rec. 150, 51-66.

Llaurado, J. G. (1958) A method for rapid adrenalectomy in the rat. F. Anim. Tech. Ass. 8, 75-78.

MAYER, G. (1963) Delayed nidation in rats: a method of exploring the mechanism of ovo-implantation. In Delayed Implantation, pp. 213-231. Ed. A. C. Enders. University of Chicago Press.

Mohla, S. \& PRASAD, M. R. N. (1970) Stimulation of RNA synthesis in the blastocyst and uterus of the rat by adenosine 3',5'-monophosphate (cyclic AMP). F. Reprod. Fert. 23, 327-329.

Posternak, Th., Sutherland, E. W. \& Henion, W. F. (1962) Derivatives of cyclic 3',5'-adenosine monophosphate. Biochim. biophys. Acta, 65, 558-560.

Rugr, R. (1968) The Mouse, its Reproduction and Development. Burgess, Minneapolis.

SHORT, R. V. (1960) The secretion of sex hormones by the adrenal gland. Biochem. Soc. Symp. 18, 59-84.

Smith, D. M. \& BigGers, J. D. (1968) The oestrogen requirement for implantation and the effect of its dose on the implantation response in the mouse. F. Endocr. 41, 1-9.

Sneld, G. D. \& Stevens, L. G. (1966) Early embryology. In Biology of the Laboratory Mouse, 2nd edn, pp. 205-245. Ed. E. L. Green. McGraw-Hill, London.

Takahashi, K. (1961) On the extra-ovarian origin of estrogen. Tohoku f. exp. Med. 75, 207-222.

WEBB, F. T. G. (1973) Extrinsic and intrinsic factors affecting implantation in mammals. D.Phil. thesis, University of Oxford. 